J. $\operatorname{CHEN}^{1,2, \square}$

A. HANGAUER ${ }^{1,2}$

R. STRZODA ${ }^{1}$

M.-C. AMANN ${ }^{2}$

\title{
Accurate extraction method for the FM response of tunable diode lasers based on wavelength modulation spectroscopy
}

\footnotetext{
${ }^{1}$ Siemens Corporate Technology, Power \& Sensor Systems, Otto-Hahn-Ring 6, 81739 Munich, Germany

${ }^{2}$ Walter Schottky Institute, Technical University of Munich, Am Coulombwall 3, 85748 Garching, Germany
}

\section{Received: 16 September 2007 \\ Published online: 8 December 2007 • C Springer-Verlag 2007}

ABSTRACT An accurate wavelength modulation spectroscopy based method to measure the dynamics of the wavelength modulation behavior of tunable diode lasers is introduced in this paper. This method requires only standard equipment and a simple mechanical setup. Under the condition of a constant laser modulation current, the wavelength modulation amplitude can be determined by analysis of the distance between the two zero crossings of the measured second harmonic spectrum of gas absorption. When measuring at different modulation frequencies, the frequency response of the current-to-wavelength tuning coefficient can be obtained. The use of a frequency analyzer instead of a lock-in amplifier to obtain the second harmonic spectrum has two advantages: it provides a higher bandwidth and allows for very precise detection of the zero crossings because of the logarithmic output. The results exhibit very good agreement with a reference measurement performed with a fast FTIR Spectrometer.

PACS 42.60.Fc; 42.62.Fi; 42.55.Fx

\section{$1 \quad$ Introduction}

Modulation of the wavelength of directly modulated tunable diode lasers can be accomplished by tuning the laser current. For small current variations, the currentto-wavelength tuning behavior is linear, therefore a small sinusoidal current variation causes a sinusoidal wavelength variation with frequency dependent amplitude attenuation and phase shift. In the following, the amplitude response of the current-to-wavelength tuning rate is denoted as 'FM response'.

In wavelength modulation spectroscopy applications, it is important to investigate the frequency dependence of the current-to-wavelength tuning coefficient so that the wavelength response can be determined from arbitrary current variation over time.

To measure a wavelength variation, a conversion of frequency/wavelength modulation (FM) to amplitude modulation (AM) is usually needed. There are several methods to measure the 'FM response' [1-3]. Usually, a Fabry-Pérot-

E-mail:jia.chen@wsi.tum.de interferometer is used for that purpose [2,4]. Another approach was presented by Schilt, who first used the change in the amplitude of the harmonic spectra in WMS to determine the FM response [1]. Since the harmonic spectra are caused by gas absorption, the FM to AM conversion is carried out by the gas absorption in this case. In this paper, the frequency dependent tuning rate is investigated by analyzing the distance between the two zero crossings of the measured second harmonic spectrum and a simple experimental setup is developed, allowing for a high speed tuning measurement. Using this method, the FM responses of different kinds of vertical-cavity surface-emitting lasers (VCSELs) [5] have been investigated.

\section{Theory and principle of the WMS measurement}

In WMS [6] the current of the laser diode is sinusoidally modulated around a bias current $\bar{I}$ with a modulation frequency $f$ and a current modulation amplitude $I_{\mathrm{a}}$ :

$I(t)=\bar{I}+I_{\mathrm{a}} \cos (2 \pi f t)$,

which causes a wavelength variation of

$\lambda(t)=\bar{\lambda}+\lambda_{\mathrm{a}} \cos (2 \pi f t+\Psi)$,

with the wavelength modulation amplitude $\lambda_{\mathrm{a}}=\lambda_{\mathrm{a}}(f)=$ $|k(f)| I_{\mathrm{a}}$ and the phase shift $\Psi=\Psi(f)=\arg (k(f))$, where $k(f)$ denotes the complex frequency dependent current-towavelength tuning coefficient. The wavelength modulated light passes through a gas which absorbs light at specific wavelengths and the resulting amplitude/intensity modulated (AM) signal is detected with a photodetector. With a lockin amplifier, the $n$-th Fourier coefficient of the AM signal at wavelength $\bar{\lambda}$ is selected. When sweeping $\bar{\lambda}$ slowly in a small range around a gas absorption line, the $n$-th harmonic components for each central wavelength $\bar{\lambda}$ form the $n$-th harmonic spectrum $S_{n}(\bar{\lambda})$.

$S_{n}(\bar{\lambda})=f \varepsilon_{n} \int_{0}^{1 / f} T(\lambda(t)) P_{L}(t) \exp (-\mathrm{i} n 2 \pi f t) \mathrm{d} t$

where $T(\lambda)$ is the transmission function that is given by gas absorption profiles according to the Beer-Lambert law and 
$P_{\mathrm{L}}(t)$ is the laser's intrinsic intensity modulation caused by the current modulation over time, which is a characteristic of the laser device and will be modeled later in this section. The factor $\varepsilon_{n}$ is given by $\varepsilon_{n}=2-\delta_{0 n}$ with the Kronecker-delta $\delta_{i j} . \varepsilon_{n}$ is 2 for $n>0$ because the lock-in amplifier selects the Fourier components both in positive and negative frequencies.

The $n$-th harmonic component is real if there is no phase shift $\Psi$ and complex otherwise. The second harmonic spectrum is therefore the second Fourier component over $\bar{\lambda}$. It is usually used for the WMS spectroscopy because there is little influence of the additional laser intensity modulation on it and less contribution from $1 / f$ noise. The width of the second harmonic spectrum given by the distance of the two zero crossings or minima not only depends on the gas temperature and pressure, but also highly on the wavelength modulation amplitude $\lambda_{\mathrm{a}}$. This effect is shown in Fig. 1 .

For the FM response, only the decrease of wavelength modulation amplitude is of interest. Therefore, it is advantageous to define a normalized correction function for the measured distance of the zero crossings.

Let $\delta \bar{\lambda}\left(\lambda_{\mathrm{a}}(f)\right)=: \delta \bar{\lambda}(f)$ be the distance of the zero crossings of the second harmonic signal at modulation frequency $f$ and $\lambda_{\mathrm{a}}(f)=|k(f)| I_{\mathrm{a}}$ the corresponding wavelength modulation amplitude. The FM response $H(f)$ is the normalized absolute value of the tuning coefficient:

$H(f)=\left|\frac{k(f)}{k(0)}\right|=\frac{\lambda_{\mathrm{a}}(f)}{\lambda_{\mathrm{a}}(0)}$.

It is expedient to normalize the distance of the zero crossings as well:

$\Delta \bar{\lambda}(f)=\frac{\delta \bar{\lambda}(f)}{\delta \bar{\lambda}(0)}$.

It does not matter in which unit the distance of zero crossings is measured, such as in time, current or wavelength, because the variables are normalized. Practically, the distance of the zero crossings at a modulation frequency near $\mathrm{dc}$ is chosen as the normalization value. The function that is used to correct the measured distance of the zero crossings shall be denoted as $F$ :

$H(f)=F(\Delta \bar{\lambda}(f))$.

This function $F$ always crosses the point $(\Delta \bar{\lambda}, H)=(1,1)$ and depends on many implicit parameters:

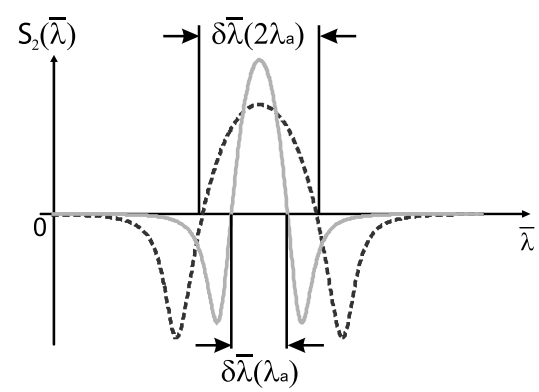

FIGURE 1 The second harmonic spectrum $(\Psi=0)$ and its dependence on the wavelength modulation amplitude, with $\delta \bar{\lambda}\left(\lambda_{\mathrm{a}}\right)$ representing the distance of the two zero crossings at modulation amplitude $\lambda_{\mathrm{a}}$
- The line parameters of the gas absorption.

- The current gas parameters (concentration, absorption path length, pressure and temperature).

- The additional AM of the laser.

- The modulation amplitude at zero frequency $\lambda_{\mathrm{a}}(0)$ or equivalently the distance of zero crossings in wavelength $\delta \bar{\lambda}(0)$.

The linearity range of the $F$ function should be large enough that the wavelength variation can still be measured even if it is very small.

Since the variation of the laser current generates an additional intensity modulation, there is another AM effect in the detector signal that also affects the shape of the harmonic spectra. This effect has to be included in the calculation of correction function $F$, thereby a model for the laser intensity $P_{\mathrm{L}}(t)$ over time with the laser injection current $I(t)$ (1) should be assumed. Three important models can be distinguished, whereby $P(I)$ denotes the laser's $P I$ characteristic measured at de condition:

- ideal model:

$$
P_{\mathrm{L}}(t)=P\left(I_{0}\right)=\text { const. }
$$

- static model:

$$
P_{\mathrm{L}}(t)=P(I(t))=P\left(\bar{I}+I_{\mathrm{a}} \cos (2 \pi f t)\right) ;
$$

- dynamic model:

$$
P_{\mathrm{L}}(t)=P\left(\bar{I}+I_{\mathrm{a}} H(f) \cos (2 \pi f t+\Psi(f))\right) .
$$

The frequency dependent phase shift $\Psi(f)$ has only a negligible contribution to the distance between zero crossings.

\section{Ideal model}

In the ideal case, the laser intensity is constant (see (7)). Arndt gives an analytical formula for the second harmonic spectrum $[7,8]$, whereas the gas absorption line must have

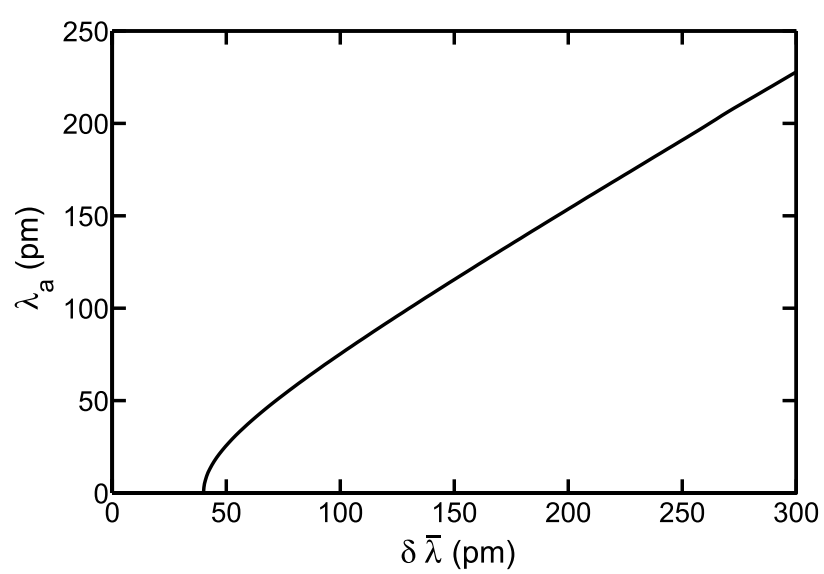

FIGURE 2 Relationship between the distance of the zero crossings $\delta \bar{\lambda}$ of the second harmonic spectrum and the wavelength modulation amplitude $\lambda_{a}$. A constant laser intensity is assumed. An $\mathrm{H}_{2} \mathrm{O}$ absorption line at $1853 \mathrm{~nm}$ with $20 \mathrm{~cm}$ path length and ambient laboratory air was used 
a Lorentz profile which is usually fulfilled under atmospheric conditions. The relationship between the distance of the zero crossings $\delta \bar{\lambda}$ and the modulation amplitude $\lambda_{\mathrm{a}}$ shown in Fig. 2 is computed by evaluating the zeros of the Arndt formula with a standard iterative algorithm. There is an almost linear correlation between $\delta \bar{\lambda}$ and $\lambda_{\mathrm{a}}$, which however does not cross the origin. It seems that the distance of the two zero crossings is limited by the half-width at half-maximum (HWHM) of the gas absorption line, which, in this case, is about $33 \mathrm{pm}$.

\section{Static model}

If it is assumed that the output power of the laser immediately follows the laser current, then the static model is used (8).

However, this model is incorrect at high frequencies because the nonlinearity of the current to intensity behavior is due to the temperature variation in the active region. With increasing frequency, the amplitude of the temperature variation decreases because heat conduction is a slow process. Thus, the nonlinearity of the intensity variations should decrease as well. This gives rise to the other model that represents the opposite extreme case as compared to the static model.

\section{Dynamic model}

For the dynamic model, it is assumed that the current to intensity behavior is dynamic and has the same dynamics as in the current to wavelength behavior (9). If the nonlinearities of the PI characteristic are only due to the internal heating of the laser, it is reasonable to assume that the frequency behavior of the nonlinearities are the same as the current-totemperature (or current-to-wavelength) behavior. This model can be simplified when the intensity scale of the $P I$ characteristic is translated to the wavelength scale. Then, the translated PI characteristic can simply be multiplied with the transmission function to simulate the effect of the laser AM on the second harmonic spectrum.

The PI characteristic of the laser causes a background distortion of the harmonic spectra [9], whereas its quadratic term causes an offset of the second harmonic spectrum that changes the distance of the zero crossings. It means that the curve representing the relationship between $\Delta \bar{\lambda}$ and $H$ will be shifted. A comparison for different models for the InP-based $1853 \mathrm{~nm}$ VCSEL [10] is shown in Fig. 3.

Generally, the linearity range of the $F$ function and the range of agreement between different models are improved by

- enlarging the ratio between the gas absorption and the background of the second harmonic. Since the nonlinearity of the laser's PI characteristic is fixed, this is accomplished by using a higher absorption line strength, gas concentration or absorption path length;

- narrowing the absorption line width (HWHM) by lowering the gas pressure;

- choosing a larger wavelength modulation amplitude near DC frequency, i.e., larger current modulation amplitude.

Here the wavelength modulation amplitude $\lambda_{\mathrm{a}}$ at the lowest frequency was six times the HWHM of the gas absorption line, while the optimum modulation amplitude for a maximum second harmonic is about twice the HWHM [7]. As can

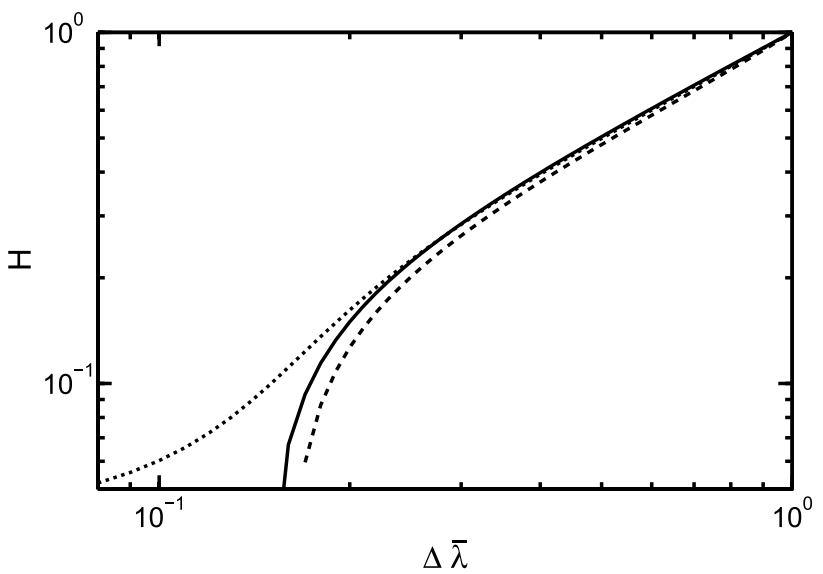

FIGURE 3 The correction function $F$ in double-logarithmic scale. The solid curve represents the simulation result of the Arndt formula (ideal model, (7)). The dotted and broken curves resemble the models of (8) and (9), respectively. An $\mathrm{H}_{2} \mathrm{O}$ absorption line at $1853 \mathrm{~nm}$ with $20 \mathrm{~cm}$ path length and ambient laboratory air was used

be estimated from Fig. 3, this allows measurement of a wavelength modulation drop up to a factor of about 5 .

For a GaAs-based 763 nm VCSEL [11], the background signal due to the quadratic term of the $P I$ characteristic is smaller than the background of the $1853 \mathrm{~nm}$ VCSEL, so that the different models behave less distinctly from each other and the measurement range is increased.

\section{Experimental setup and characterization of the FM response of VCSELS}

\subsection{Measurement with WMS}

The measurement setup, as shown in Fig. 4, is the basic setup for a WMS system measuring a second harmonic spectrum apart from the fact that a frequency analyzer is used instead of a lock-in amplifier and that the laser diode is driven directly by the function generator. The internal clock frequency of the function generator and the internal clock of the frequency analyzer have to be synchronized. Two function generators are used to produce a ramp and a sinusoidal voltage which are added by a simple analog circuit. Thus, the wavelength of the laser is varied sinusoidally around a center wavelength which is swept slowly with a frequency of

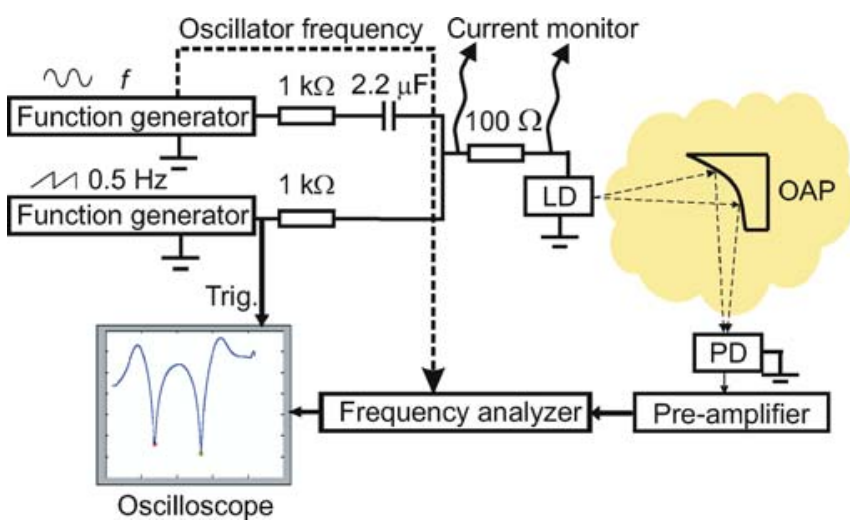

FIGURE 4 Experimental setup for measuring the FM response with the WMS method. LD: laserdiode, PD: photodiode, OAP: off axis parabola 
$0.5 \mathrm{~Hz}$. Current offset and ramp amplitude are chosen so that the second harmonic spectrum is centered and fills the display. Because of bandwidth limitations, a commercial laser controller is not used to control the laser current. Instead, the laser chip is connected to the function generators directly by an additional shunt resistance of $100 \Omega$ to monitor the laser current. The heat sink temperature of the laser chip is kept constant with a temperature controller (Profile LDC 402B).

An off axis parabola (OAP) is used to focus the light onto the detector and to extend the path length so that the signal-tonoise ratio is increased and the interference fringes between the laser and the detector are minimized. The pre-amplifier (Hamamatsu C6438) used has a cutoff frequency of $2 \mathrm{MHz}$ at a gain of $10^{3} \mathrm{~V} / \mathrm{A}$. The frequency analyzer (HP 3585A) measures a single point at twice the modulation frequency $f$, for which its internal frequency sweep has to be turned off. The resolution bandwidth is set to $10 \mathrm{~Hz}$ to enable a very sensitive measurement. Therefore, the synchronization of the oscillator frequency becomes very important. It is a notable advantage that the frequency analyzer outputs the logarithm of the absolute value of the second harmonic spectrum $\log \left|S_{2}(\bar{\lambda})\right|$ because the two zero crossings are transformed into two very easily detectable sharp peaks as shown in Fig. 5. It is also possible to determine the maximum and minima of the second harmonic spectrum which correspond to the primary and secondary maximum of the signal of the frequency analyzer. The distance of the two minima as well as the ratio between the maximum and minimum could be also taken as a measure for the modulation amplitude $\lambda_{\mathrm{a}}$ as well. The minima are not sharp peaks like zero crossings on a logarithmic scale and are therefore not detectable with a great deal of precision. The amplitude of the detector signal always depends on the bandwidth of the measuring equipment such as the pre-amplifier, which is the reason for the upper frequency limit in Schilt measurement [1]. The measurement of a time distance is, in principle, insensitive to bandwidth and noise issues.

Although in theory any harmonic spectrum may be chosen, the second harmonic was selected. The influence of the laser AM on the second harmonic spectrum is small enough compared to the first harmonic, while the amplitude of the sec-

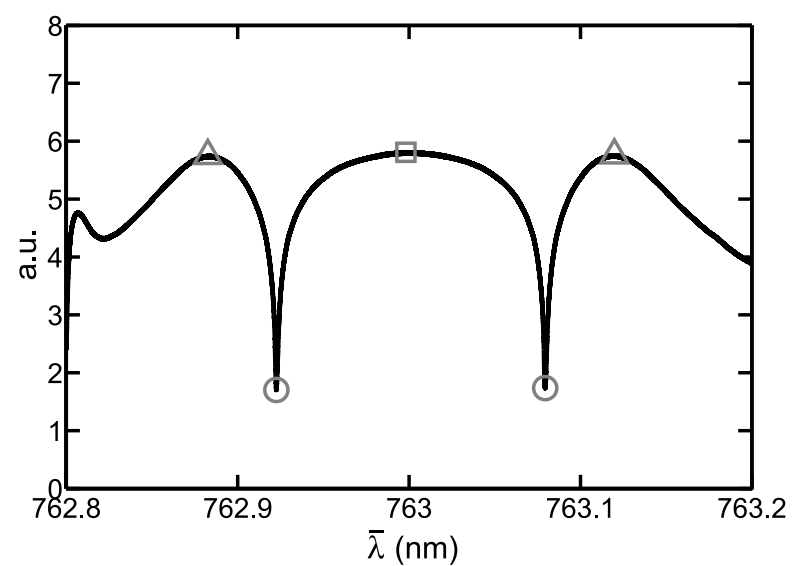

FIGURE 5 The second harmonic spectrum when measured with a frequency analyzer: $\log \left|S_{2}(\bar{\lambda})\right|$. The characteristic points are the maximum (square), the two minima (triangles) and the zero crossings (circles) of the harmonic spectrum ond harmonic is still relatively large compared to the higher harmonics.

\subsection{Measurement with a step scan spectrometer}

To guarantee the correctness of the new method, reference measurements with a step scan spectrometer (Bruker Vertex 70) were carried out. The laser was modulated with a sinusoidal current at frequency $f$ with the same amplitude as in the WMS measurement. The temperature of the laser was also stabilized with a temperature controller. A step scan spectrometer uses a short time fourier transform (STFT) to get the momentary spectrum of the light at certain short time intervals. The time intervals were shifted until at least one period of the modulation signal (i.e., $T_{0}=1 / f$ ) was covered. The emission peak of the spectrum taken at each interval is detected and taken as the momentary wavelength of the laser diode. With sinusoidal injection current, a sinusoidal variation of the emission peak is expected and practically observed. The wavelength modulation amplitude is then determined with a curve fit.

With increasing modulation frequency, the wavelength modulation amplitude decreases. The factor of decrease of the wavelength amplitude at different frequencies results in the FM response.

\subsection{Measurement procedure}

To summarize, the measurement procedure works as follows:

- Select a gas absorption line in the tuning range of the laser which gives a strong enough absorption so that the offset of the second harmonic spectrum due to the laser PI characteristic is minimized.

- Measure the second harmonic spectrum at different modulation frequencies with a spectrum analyzer instead of a lock-in amplifier.

- Detect the distance of the two sharp peaks (zero crossings) off-line.

- Normalize the distances with the distance of the zero crossings at a near dc frequency.

- Use the correction function $F$ to get the normalized FM response.

\subsection{Measurement data of the FM response}

In Fig. 6, the measurement data for the InP-based $1853 \mathrm{~nm}$ VCSEL from the WMS method and step scan spectrometer are shown. Both methods show very good agreement. Due to the automatic control of the WMS measurement equipment, it was possible to take more samples around the cutoff frequency (about $100 \mathrm{kHz}$ ) and to measure the FM response up to at least $1 \mathrm{MHz}$. Compared to the spectrometer measurement, the correction with the ideal model gave a maximum error of $6 \%$, the dynamic model $8 \%$ and the static model $5 \%$. Since all the models describe extreme cases, the correction should be good enough to approximate the real case. It is important to note that these error values not only depend on the PI model, but also strongly on the strength of the gas absorption. In Fig. 6, the static model is used for the correction. 


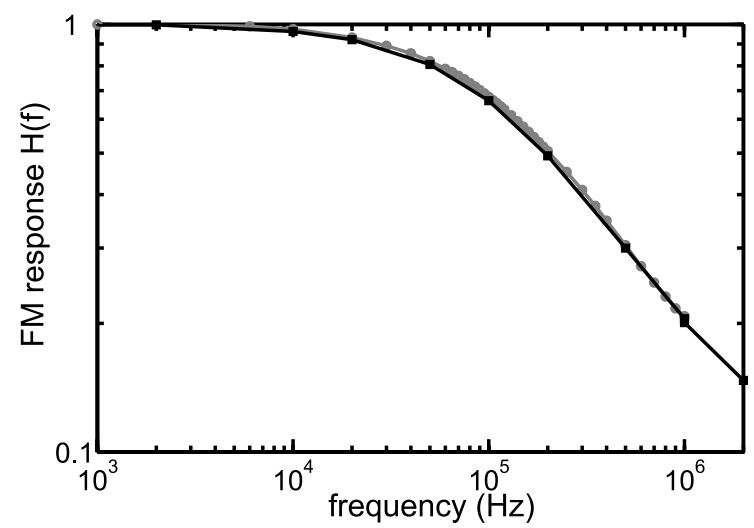

FIGURE 6 The measurement data of FM response of a InP-based $1853 \mathrm{~nm}$ VCSEL using the WMS method (gray curve) and spectrometer (black curve)

The measured FM responses of VCSELs exhibit no typical first order low-pass behavior, but follow a square root law [12]. To determine the cutoff frequency, step response measurements with an interferometer are therefore not suitable or difficult because the laser's step response is completely different from an exponential function.

\section{4}

\section{Conclusion}

In this paper, an accurate extraction method using wavelength modulation spectroscopy is introduced that measures the FM response of a laser using a gas absorption line. Absorption lines of ambient gases at atmospheric conditions were used and thus the additional complexity of a gas cell was avoided. The distance of the zero crossings of the second harmonic spectrum is detected for each modulation frequency for a constant modulation current amplitude. The correction function to compute the FM response $H(f)$ from the normalized distance of zero crossings $\Delta \bar{\lambda}$ was investigated. For this purpose, the Arndt formula can be directly used if the second harmonic spectrum is large enough compared to the back- ground signal due to the nonlinearity of the $P I$ characteristic of a laser. To estimate the error due to the PI characteristic, two different models which describe extreme cases have been developed. The measurement range is determined by the agreement between the models (Fig. 3), which is dependent on the current modulation amplitude and the ratio between the background signal and the absorption. Here it was possible to measure up to a wavelength amplitude drop factor of about 5 , which in this case corresponds to a frequency of $1 \mathrm{MHz}$. The measurement range is enhanced for lasers with low PI nonlinearities or if a stronger gas absorption, lower gas pressure or higher current modulation amplitude is used.

A reference measurement with a step scan spectrometer was carried out, which validates the WMS measurement. The WMS method requires only standard equipment and a simple mechanical setup compared to an interferometer measurement.

ACKNOWLEDGEMENTS The authors gratefully acknowledge the financial support provided by the Federal Ministry of Education and Research of Germany (Project 'NOSE', contract No. 13N8772) and wish to thank Andreas Goedecke for the valuable comments to this article.

\section{REFERENCES}

1 S. Schilt, L. Thévenaz, Appl. Opt. 43, 4446 (2004)

2 A. Lytkine, W. Jäger, J. Tulip, Spectrochim. Acta A 63, 940 (2006)

3 A. Lytkine, W. Jäger, J. Tulip, Proc. SPIE 5737, 157 (2005)

4 G. Hernandez, Fabry-Pérot Interferometers (Cambridge University Press, Cambridge, 1986)

5 H. Li, K. Iga, Vertical-Cavity Surface-Emitting Laser Devices (Springer, Berlin, 2002)

6 J.M. Supplee, E.A. Whittaker, W. Lenth, Appl. Opt. 33, 6294 (1994)

7 R. Arndt, J. Appl. Phys. 36, 2522 (1965)

8 S. Schilt, L. Thévenaz, P. Robert, Appl. Opt. 42, 6728 (2003)

9 P. Kluczynski, O. Axner, Appl. Opt. 38, 5803 (1999)

10 M.-C. Amann, M. Ortsiefer, Phys. Stat. Solidi A 203, 3538 (2006)

11 M. Grabherr, D. Wiedenmann, R. Jäger, R. King, Proc. SPIE 5737, 120 (2005)

12 J. Chen, A. Hangauer, R. Strzoda, M.-C. Amann, Appl. Phys. Lett. 91, (2007) (published online, DOI: 10.1063/1.2794406) 